\title{
Who is this gene and what does it do? A toolkit for munging transcriptomics data in python
}

\author{
Charles K. Fisher,* Aaron M. Smith, and Jonathan R. Walsh \\ Unlearn.AI, Inc., San Francisco, CA 94108
}

(Dated: April 5, 2018)

\begin{abstract}
Transcriptional regulation is extremely complicated. Unfortunately, so is working with transcriptional data. Genes can be referred to using a multitude of different identifiers and are assigned to an ever increasing number of categories. Gene expression data may be available in a variety of units (e.g, counts, RPKMs, TPMs). Batch effects dominate signal, but metadata may not be available. Most of the tools are written in R. Here, we introduce a library, genemunge, that makes it easier to work with transcriptional data in python. This includes translating between various types of gene names, accessing Gene Ontology (GO) information, obtaining expression levels of genes in healthy tissue, correcting for batch effects, and using prior knowledge to select sets of genes for further analysis. Code for genemunge is freely available on Github.
\end{abstract}

\footnotetext{
*drckf@unlearn.ai; authors listed alphabetically.
} 


\section{OVERVIEW}

munge: verb

1. to manipulate (raw data), especially

to convert (data) from one format to

another.

www.dictionary.com/browse/munge

Like any area that uses big data, transcriptomics data requires extensive munging - rote but critical tasks such as cleaning data, selecting relevant data, structuring metadata, and making labels interpretable. These tasks often need to be repeated on a given project as the data and aims evolve, and tend to be similar between different analyses. To face these challenges, a library of data munging tools can be extraordinarily useful. Such a library can provide reliable and tested tools to cleanly separate munging tasks from analysis, making it easier to start new projects and data processing pipelines less fragile.

This note introduces genemunge, a library of tools for working with human transcriptomics data. genemunge is written in python and is available as a package through PyPI. This initial version, v0.0, contains tools for tasks such as:

- Translating between conventions for gene symbols [1].

- Accessing Gene Ontology (GO) metadata [2-4].

- Using prior knowledge of biological and molecular processes to select gene sets [5, 6].

- Retrieving statistics on gene expression in healthy tissue [7-10].

- Converting expression data to TPM from counts or RPKM [11].

- Correcting for unobserved, and uninteresting, factors of variation (i.e., batch effects). $[12,13]$.

The goal of genemunge, and its current use case for the authors, is to serve as a resource for gene information that can return useful data structures and be integrated into processing pipelines. The next section gives a few example use cases of the library. 


\section{EXAMPLE USE CASES OF GENEMUNGE}

We consider a simple analysis where genemunge is useful. Suppose we want to find genes associated with the immune system, select those with larger expression in the small intestine than the stomach, and then retrieve basic information about those genes.

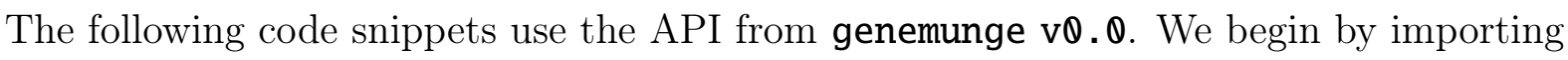
libraries required for the example.

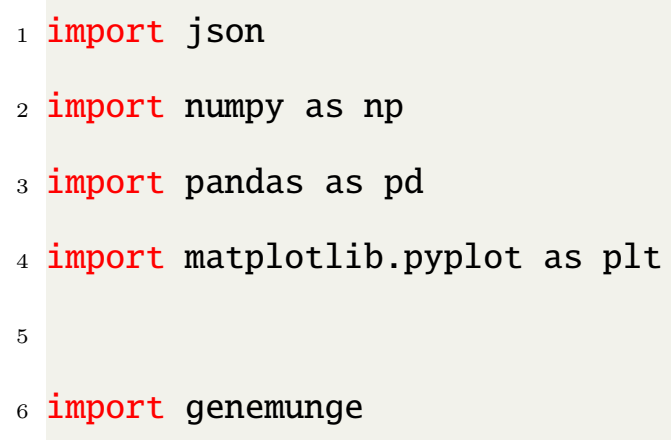

\section{A. Searching the gene ontology for relevant genes}

The Gene Ontology (GO) contains basic descriptors for each ontology entry [2-4]. Since we want genes related to the immune system, we will do a keyword search for immune and retrieve GO identifiers with this keyword. We can then obtain the associated genes.

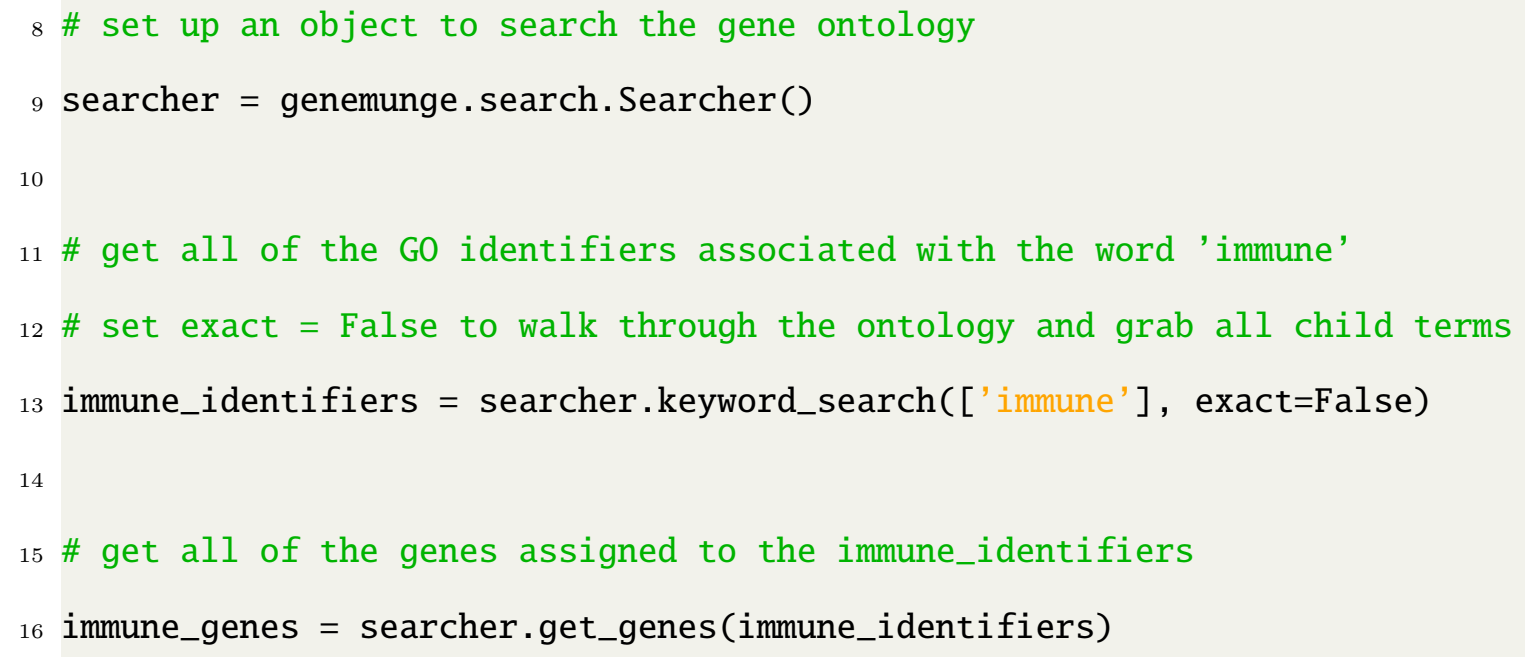

We will then use prior knowledge to remove housekeeping genes. A list of housekeeping genes curated by [6] is stored in genemunge. 
18 \# get a list of housekeeping genes

19 housekeeping = searcher.get_housekeeping_genes()

20

21 \# keep all of the immune related genes that are not housekeeping genes

22 variable_immune_genes $=$ list(set(immune_genes) - set(housekeeping))

\section{B. Obtaining statistics about gene expression}

We can use genemunge to access summary statistics from the GTEx project (through recount) about expression levels in healthy tissue [7-10]. The median expression value can be used to find genes that are more expressed in the small intestine than the stomach.

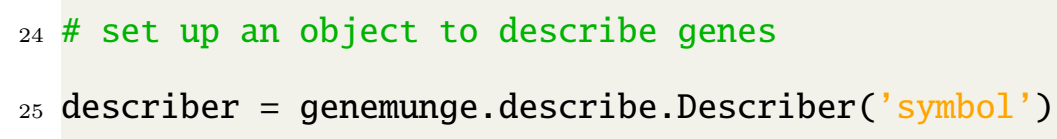

26 27 \# find the absolute and relative expression levels of each gene of interest 28 expression_data $=$ pd.DataFrame $($ index=variable_immune_genes, columns $=[$ 'expression', 'log ratio'])

30 31 \# get the expression levels in healthy tissue (in TPM units)

32 stats = describer.tissue_stats['median'].reindex(variable_immune_genes) 33 expression_data['expression'] = stats['Small Intestine']

34 35 \# control the log with a small pseudocount

36 pseudocount $=1.0$

37 expression_data['log ratio'] = np. $\log 10($

38 (pseudocount + stats['Small Intestine']) / (pseudocount + stats['Stomach']))

A scatter plot of the relative expression in the small intestine to the stomach against the absolute expression in the small intestine is shown in Figure 1.

40 \# plot the gene expression fraction

$41 \mathrm{fig}, \mathrm{ax}=$ plt.subplots $($ figsize $=(12,8))$ 
42 plt.xticks (fontsize $=16$ )

43 plt.yticks (fontsize=16)

44

45 ax.scatter(expression_data['expression'], expression_data['log ratio'])

46 ax.set_xlabel('Small Intestine expression [TPM]', fontsize=20)

47 ax.set_ylabel ('log10 ratio (Small Intestine / Stomach)', fontsize=20)

48 ax.set_xscale('log')

49 ax.set_xlim([0.001,1e5])

50 ax.set_ylim( $[-3,4])$

51 plt.savefig('small_intestine_example.png', bbox_inches='tight', dpi=300)

52 plt.show()

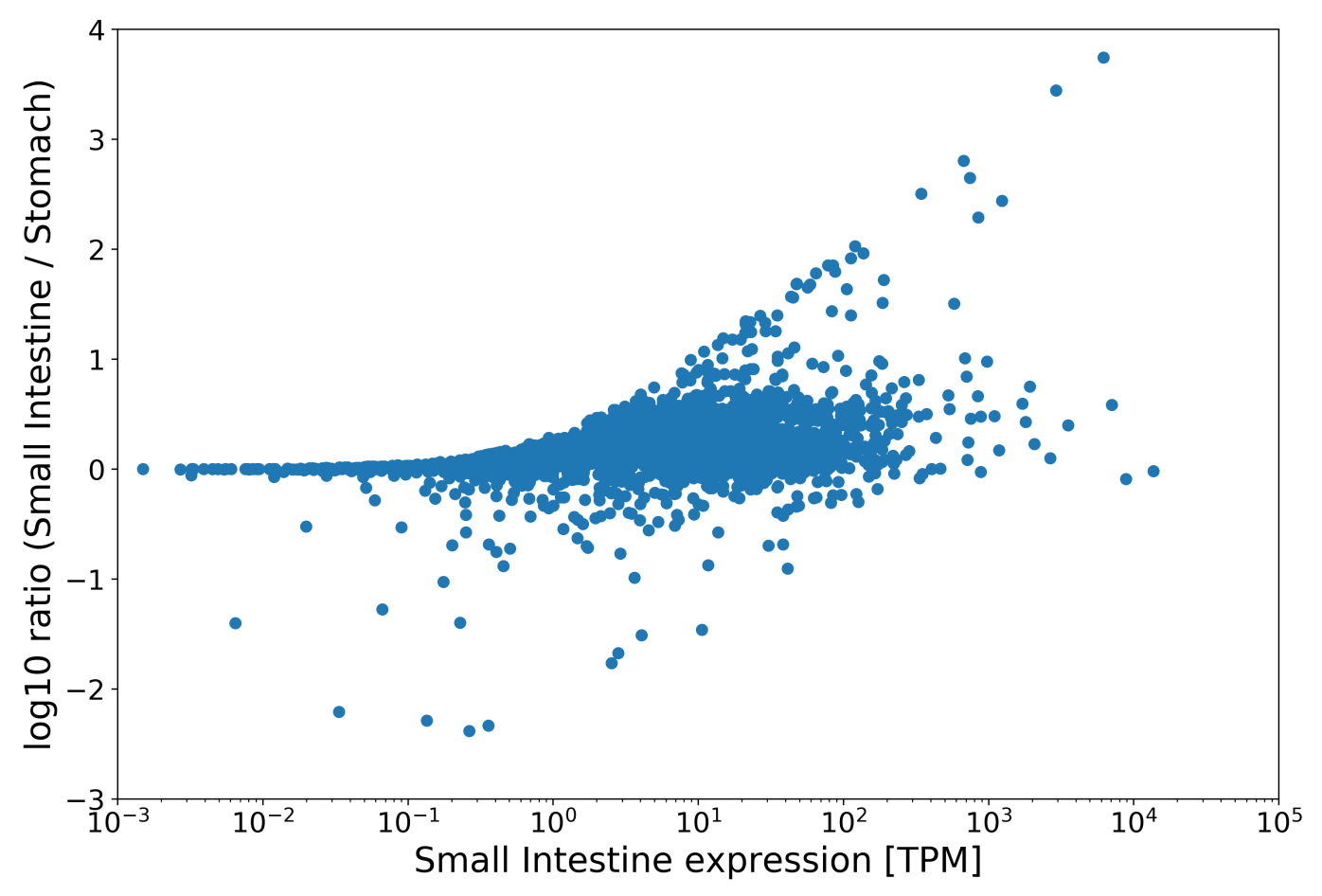

FIG. 1: Scatter plot showing the relative expression between the small intestine and the stomach versus the absolute expression in the small intestine. Genes of interest are in the upper arm. 


\section{Converting between gene identifier types}

In genemunge, the base representation of genes is in terms of their Ensembl ID (without a version number). We will want to see the gene symbol in the results, so we convert the gene symbols.

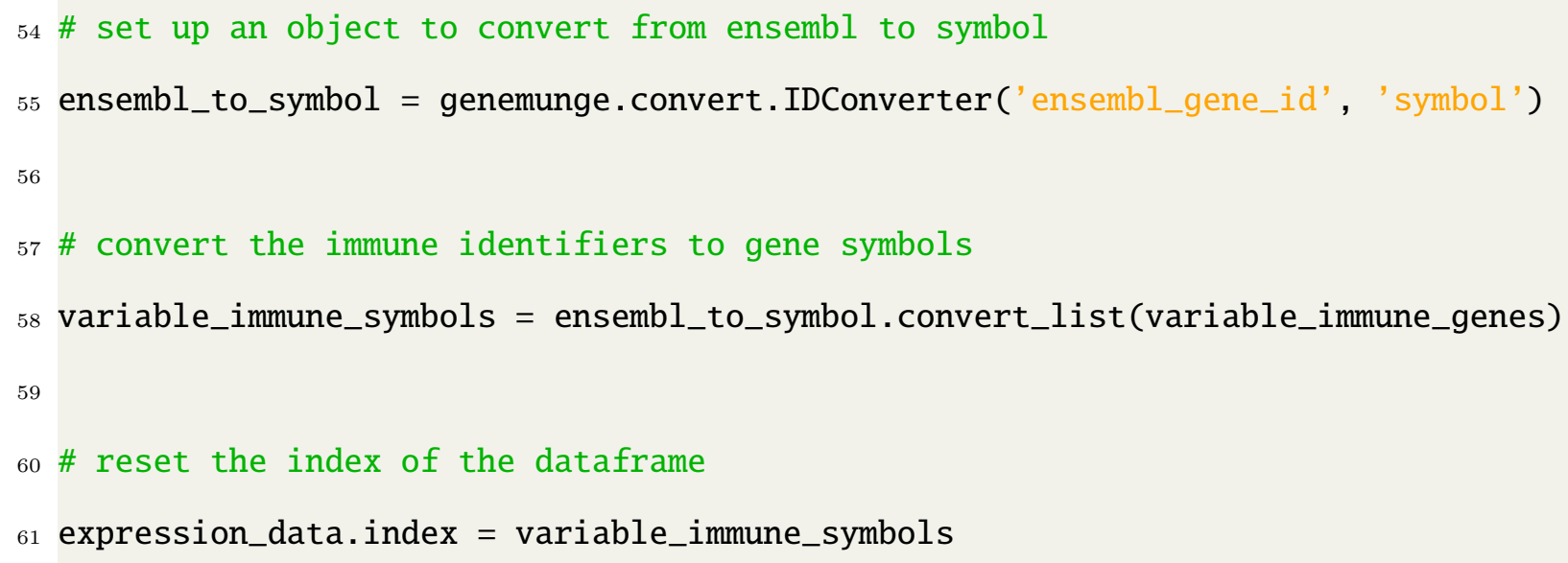

We can then select genes with high relative expression. Of course, one should be careful with this type of thing and do a differential expression analysis, but we'll just wing it.

63 \# select genes with high relative expression

64 target_genes = expression_data[expression_data['log ratio'] > 1]

65 target_genes $=$ target_genes.sort_values (by=['expression.' , ascending=False)

\section{Displaying information about a gene}

Finally, we can look at metadata and gene expression data from GTEx on one of these genes (in this case, the most highly expressed gene).

67 \# get some basic information about one of the immune genes

68 print (json.dumps(describer.get_gene_info(target_genes.index $[0]$ ), indent $=2$ ))

69

70 \# make a plot of the expression of one of the immune genes across tissues

71 describer.plot_tissue_expression(target_genes.index[0], sortby='median',

The genemunge output of the gene info is

"ensembl": "ENSG00000164816", 


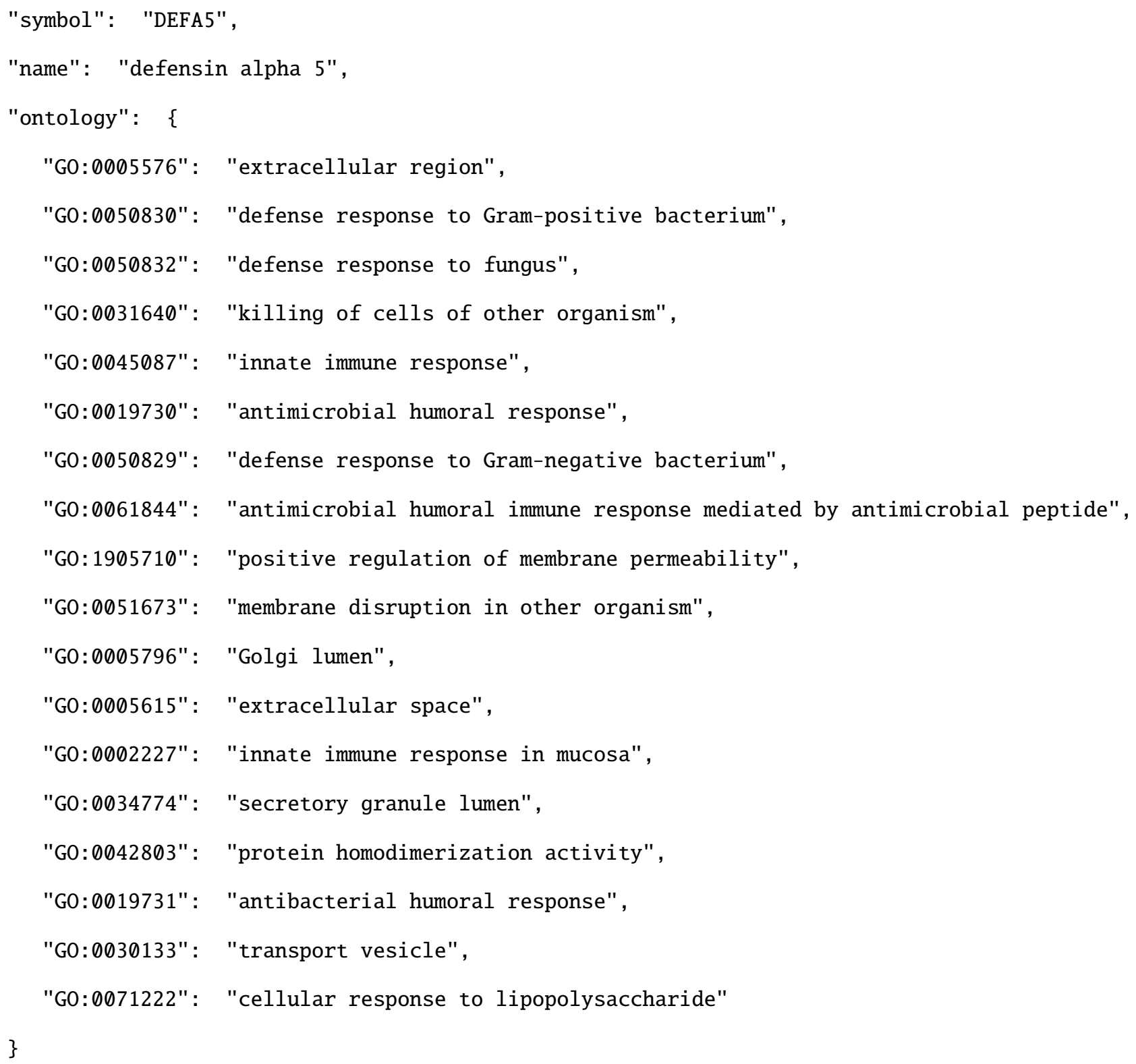

and the gene expression profile from GTEx is shown in Figure 2. As expected, DEFA5 is highly expressed in the small intestine and, in this case, nowhere else.

\section{E. Other stuff we aren't covering}

This example does not use all of the features of genemunge. For example, one could also use genemunge to identify transcription factors, or look up the lengths of genes and use them to convert data from counts to TPM units. You can even perform unsupervised correction of batch effects using the Remove Unwanted Variation (RUV) algorithm [12, 13]. You can read more about all of the amazing things genemunge can do in the documentation. 


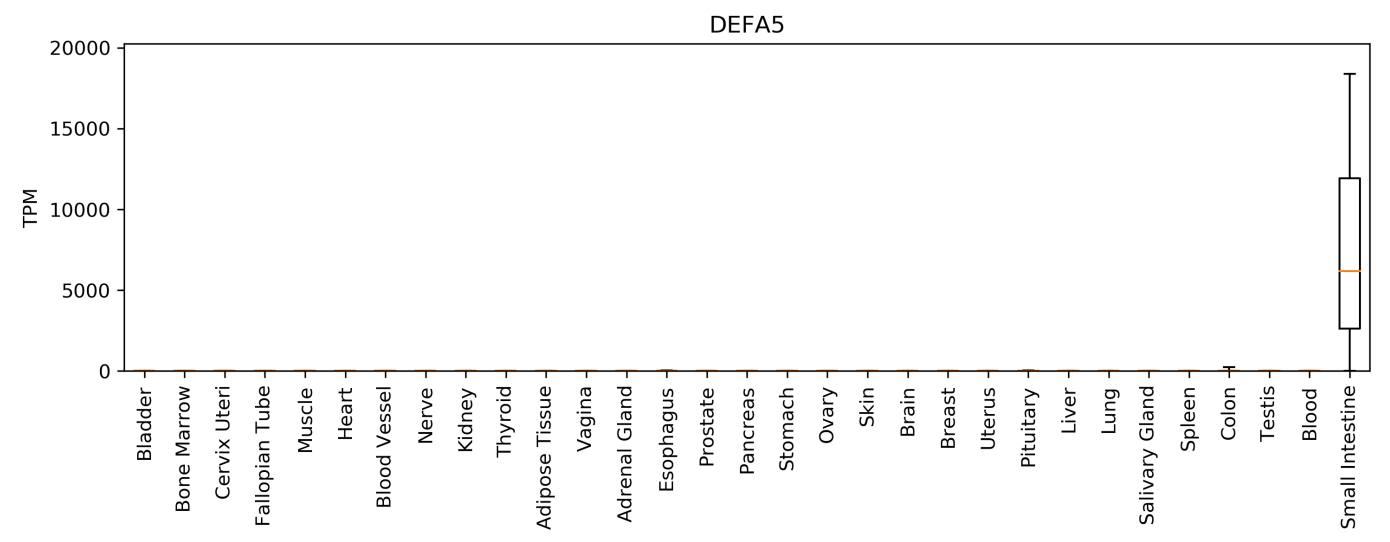

FIG. 2: Gene expression profile in healthy tissue from GTEx for an example gene.

\section{SUMMARY}

genemunge was built to make working with transcriptomics data easier. It provides a simple way to select genes of interest in an analysis and return useful metadata about them. We find that it is a useful component of a larger analysis and data processing pipeline. Our intent on open sourcing the package is to engage with the computational biology community and build it into a broadly useful tool. We welcome feedback, feature requests, and contributions on genemunge through GitHub.

[1] E. A. Bruford, M. J. Lush, M. W. Wright, T. P. Sneddon, S. Povey, and E. Birney, Nucleic acids research 36, D445 (2007).

[2] M. Ashburner, C. A. Ball, J. A. Blake, D. Botstein, H. Butler, J. M. Cherry, A. P. Davis, K. Dolinski, S. S. Dwight, J. T. Eppig, et al., Nature genetics 25, 25 (2000).

[3] G. O. Consortium, Nucleic acids research 32, D258 (2004).

[4] S. Carbon, A. Ireland, C. J. Mungall, S. Shu, B. Marshall, S. Lewis, A. Hub, and W. P. W. Group, Bioinformatics 25, 288 (2008).

[5] K. Chawla, S. Tripathi, L. Thommesen, A. Lægreid, and M. Kuiper, Bioinformatics 29, 2519 (2013).

[6] E. Eisenberg and E. Y. Levanon, Trends in Genetics 29, 569 (2013).

[7] L. J. Carithers, K. Ardlie, M. Barcus, P. A. Branton, A. Britton, S. A. Buia, C. C. Compton, D. S. DeLuca, J. Peter-Demchok, E. T. Gelfand, et al., Biopreservation and biobanking 13, 
311 (2015).

[8] L. J. Carithers and H. M. Moore, "The genotype-tissue expression (gtex) project," (2015).

[9] A. C. Frazee, B. Langmead, and J. T. Leek, BMC bioinformatics 12, 449 (2011).

[10] L. Collado-Torres, A. Nellore, K. Kammers, S. E. Ellis, M. A. Taub, K. D. Hansen, A. E. Jaffe, B. Langmead, and J. T. Leek, Nature biotechnology 35, 319 (2017).

[11] G. P. Wagner, K. Kin, and V. J. Lynch, Theory in biosciences 131, 281 (2012).

[12] J. A. Gagnon-Bartsch and T. P. Speed, Biostatistics 13, 539 (2012).

[13] L. Jacob, J. A. Gagnon-Bartsch, and T. P. Speed, Biostatistics 17, 16 (2015). 